\title{
The feasibility of lengthening sleep in habitually short sleepers and its effect on dietary intake, energy balance, and metabolic risk factors: A randomised controlled trial
}

\author{
H.K. Al Khatib ${ }^{1}$, W.L. Hall ${ }^{1}$, A. Creedon ${ }^{1}$, E. Ooi ${ }^{1}$, T. Masri ${ }^{1}$, S.V. Harding ${ }^{1}$, L. McGowan ${ }^{2}$, \\ J. Darzi ${ }^{1 *}$ and G.K. Pot ${ }^{1,3 *}$ \\ ${ }^{1}$ Diabetes \& Nutritional Sciences Division, Faculty of Life Sciences \& Medicine, King's College London, \\ London SE1 9NH, U.K., \\ ${ }^{2}$ Centre for Public Health, School of Medicine, Dentistry and Biomedical Sciences, Queens University Belfast, \\ Belfast BT12 6BA, U.K. and \\ ${ }^{3}$ Department of Health and Life, Virje Universiteit Amsterdam, Faculty of Earth and Life Sciences, Amsterdam,
} The Netherlands

Sleep is an unquestionable contributor to optimal health. The current guidelines for adults aged 18-64 years recommend 7-9 hours of sleep per night for physical and mental well-being ${ }^{(1)}$. Present patterns of sleep duration diverge from these recommendations due to lifestyle demands of the modern society ${ }^{(2)}$. The reduction in sleep duration has been paralleled by the increased prevalence in obesity and metabolic diseases over the past decades ${ }^{(3)}$. Intervention studies have demonstrated sleep deprivation may causally induce metabolic dysregulation $^{(4)}$ and positive energy balance ${ }^{(5)}$, driving the incidence of obesity and metabolic diseases. However, few studies have examined sleep's putative therapeutic impacts.

To the best of our knowledge, this is one of the first randomised controlled trials (RCT) in healthy, free-living non-obese adults to attempt to lengthen sleep over an extended period, and uses objective measures of sleep to confirm compliance. In a parallel design, we investigated the feasibility of extending sleep using behavioural change techniques targeting sleep hygiene over a four week intervention period in habitually short sleepers (5-<7 hours/night) (Trial Registration: NCT02787577). The intervention group received a personalised sleep hygiene consultation session to extend their time in bed by $1-1.5$ hours, and the control group were asked to resume their lifestyle as normal. Eligible participants were healthy men and women, aged 18-64 years, and were screened for sleep disorders and risk of low mood. The control and intervention groups were matched for age, sex, BMI and ethnicity by randomising with minimisation. Objective measures of sleep using 7-day wrist actigraphy (MotionWatch8, CamNtech Ltd, Cambridge, UK) were recorded at baseline and endpoint. We also collected pilot data to investigate possible effects on components of energy balance and metabolic risk indicators and to provide data for calculating the required sample size for a full trial in the future, including 7-day food diaries, energy expenditure, anthropometric measures, fasting blood samples, and blood pressure. A total of 43 participants (control $\mathrm{n}=21$, intervention $\mathrm{n}=22$ ) completed the study.

\begin{tabular}{|c|c|c|c|c|c|}
\hline \multirow[b]{2}{*}{ (Minutes) } & \multicolumn{2}{|l|}{ Control } & \multicolumn{2}{|l|}{ Intervention } & \multirow[b]{2}{*}{ Mean difference at endpoint } \\
\hline & Baseline & Endpoint & Baseline & Endpoint & \\
\hline Time in Bed & $429(411,448)$ & $426(407,445)^{*}$ & $423(405,441)$ & $476(456,496)^{*}$ & $56(31,81)$ \\
\hline Assumed Sleep & $414(396,432)$ & $408(389,427)^{*}$ & $410(393,428)$ & $455(436,474)^{*}$ & $52(27,77)$ \\
\hline Actual Sleep & $353(336,371)$ & $346(328,364)^{*}$ & $363(348,379)$ & $379(364,394)^{*}$ & $32(11,54)$ \\
\hline
\end{tabular}

Values are reported as mean $(95 \% \mathrm{CI})$. Difference between groups at endpoint tested by ANCOVA, with baseline measurements as a covariate. Values presented at endpoint are estimated marginal means.

* Difference between groups statistically significant, $P<0.01$.

All measures of sleep duration increased in the intervention group in comparison to the control group, confirming the efficacy of the behavioural change intervention strategy. We found no significant difference in all indicators of healthy lifestyle measures obtained. However, we found trends toward reduced carbohydrate (Mean $\Delta-19.5 \mathrm{~g}, 95 \% \mathrm{CI} 3 \cdot 5,-42 \cdot 0, P=0 \cdot 09$ ) and free sugars intakes (Mean $\Delta-6.8 \mathrm{~g}, 95 \% \mathrm{CI}-14.9,1.3, P=0.06$ ) in the intervention group compared to control. This feasibility study was likely to be statistically underpowered to detect differences in reported dietary intakes but data will be used to plan a larger fully powered trial using this behavioural sleep strategy to investigate the impact of sleep on free-living dietary intake. Current recommendations for weight and metabolic disease management may benefit from the addition of advice on sleep hygiene.

1. Hirshkowitz M, Whiton K, Albert SM et al. (2015) Sleep Health 1, 40-3.

2. Shochat T (2012) Nat Sci Sleep 4, 19-31.

3. Schmid SM, Hallschmid M, Schultes B (2015) Lancet Diabetes Endocrinol 3, 52-62.

4. Nedeltcheva AV, Kessler L, Imperial J et al. (2009) J Clin Endocrinol Metab 94, 3242-50.

5. Al Khatib H, Harding SV, Darzi J et al. (2016) Eur J Clin, 1-11.

*contributed equally 